\title{
Fabienne BLAISE \\ « UNE POLÉMIQUE TRAGIQUE : LE SECOND VOLET DE L'AJAX DE SOPHOCLE 》
}

\author{
[dans : Revue des Etudes Grecques 112, 1999/2, p. 383-408]
}

\begin{abstract}
[383] RESUME. - Loin d'être, comme on l'a souvent pensé, une discussion mal venue dans une tragédie qui aurait pu se clore sur le suicide d'Ajax, le conflit soulevé par l'ensevelissement du héros redouble le tragique de la pièce. Le drame individuel se double d'un drame collectif en révélant une société dont le discours ne parvient pas à intégrer de manière adéquate la nature du héros qu'elle va pourtant honorer. Les propos inefficaces des Atrides montrent que l'autorité traditionnelle, qu'elle repose sur la peur ou sur une justice toute apparente, ne peut rendre compte d'une individualité hors norme mais bien réelle, et digne d'être reconnue. Leur identité de chefs est elle-même sérieusement entamée par cet échec. Ulysse résout l'aporie au prix d'une relativisation de toutes les valeurs qui n'apporte au problème particulier posé par Ajax qu'une solution formelle. Ajax, lui, meurt une seconde fois, car l'honneur qui lui est concédé nie tout ce pour quoi il est mort. Cette gloire n'est pas le fruit d'un consensus naturel, et elle lui est accordée par son pire ennemi, qui la fonde sur l'acceptation de l'altérabilité et de la légèreté humaine : or Ajax s'était suicidé précisément parce qu'il refusait une telle conception du monde. L'apparent apaisement final parvient donc mal à cacher que le désastre est complet.
\end{abstract}

ABSTRACT. - The dispute over Ajax's burial has wrongly been criticized as irrelevant and undramatic. In fact, it increases the tragic of the play, because a collective drama is added to the individual one : the nature of the hero does not succeed in being really integrated by a society which will however give him a cult. The verbal struggle leads to a total loss. The Atridae's useless words show that the traditional authority, whether it is founded on fear or on an apparent justice, is not able to assume an individuality which is abnormal but very actual and worthy of recognition, so that their credibility and their identity are seriously spoiled by this check. The restoration of Ajax's honour by Odysseus is based on completely relativistic values and is just a formal reply to the specific problem which is set by the huge hero. As for Ajax himself, the kleos which is given to [384] him is the contrary of all that for the sake of which he is dead. This glory is not the result of a natural consensus, but it is conceded to him by his worst enemy, who grounds it on the acceptance of an idea always rejected by him : human nothingness. Therefore, the apparent peace which closes the tragedy does not hide the completeness of the disaster.

Le problème que pose la seconde partie de l'Ajax a été une question si souvent débattue qu'il pourrait a priori sembler superflu de l'exhumer. À la lecture que la plupart ont donnée du dernier tiers de la pièce, ce qui frappe pourtant est la rapidité des jugements portés sur le débat serré qui suit le suicide du héros. Aveuglés par le rayonnement d'un Ajax auto-sacrifié et d'un Ulysse miséricordieux, choqués par les invectives des Atrides et de Teucros, qui semblaient contraires à la décence qu'impose la présence d'un mort, les commentateurs se sont bien souvent arrêtés à ces impressions puissantes pour donner à peu près tous une lecture en surface de cette dernière section. On ne prête guère attention aux propos des uns et des autres, dans lesquels on se contente de retrouver les signes de l'appréciation que l'on porte sur le personnage qui les tient. Les jugements de valeur font office d'interprétation, et trahissent en réalité, malgré tous les efforts de justification, la permanence du malaise que provoque le passage : on aurait préféré que la tragédie se referme sur la mort si poignante d'Ajax, que l'émotion honore de son silence le héros immolé.

Les scolies antiques condamnaient déjà, et sans détours, la longue discussion qui oppose les Atrides à Teucros, puis à Ulysse : elle rompait désagréablement avec l'idée que I'on semblait se faire du tragique en en détruisant le pathos ${ }^{1}$. Le

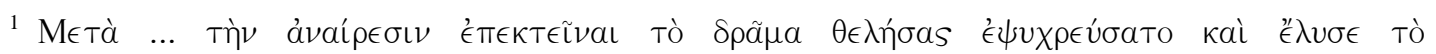


passage n'a pas moins étonné et déçu le lecteur « moderne » ${ }^{2}$. À la critique déjà formulée par la scolie à propos d'une pièce qui perdait de sa puissance en se prolongeant après l'ultime action d'Ajax ${ }^{3}$ s'en ajoutait une autre : la joute verbale ne répondait pas au goût actuel et risquait donc de priver l'Ajax du label d'intemporalité que se devait de mériter une bonne tragédie classique. La [385] longueur et la violence des altercations pouvaient en effet plaire à un public athénien amateur de débats judiciaires, mais choquaient le commentateur moderne, beaucoup moins intéressé de surcroît que les auditeurs de Sophocle par les problèmes de funérailles ${ }^{4}$.

La pièce nous étant parvenue en l'état, il fallait bien pourtant essayer de comprendre ce que pouvait apporter une si longue conclusion. Rares sont les critiques qui affirment comme Jebb que « la décision qui porte sur l'ensevelissement est la véritable climax du drame $\gg^{5}$, mais la plupart semblent considérer que l'on quitte le tragique proprement dit, qui a culminé dans le suicide, pour une série de scènes plus proches, formellement, de l'agôn tel que peut le pratiquer la comédie ${ }^{6}$. Dans les deux cas, on donne pour fonction au dernier tiers de la pièce de réhabiliter le héros. On obéit en fait ainsi à une nécessité qui peut être de deux ordres. Esthétique, en quelque sorte, lorsque I'on considère qu'Ajax, dans son refus des limites que le religieux impose, incarne la figure du héros qui excède l'humain. Condamné par là même à la destruction, il n'en reste pas moins un colosse dont la pièce doit jusqu'au bout exhiber la grandeur ${ }^{7}$. On retrouve ce sentiment chez la plupart des interprètes, mais il prend souvent appui sur le rappel d'une réalité historique. L'Athènes du $V^{\mathrm{e}}$ siècle honorait Ajax d'un culte héroïque. Il fallait donc que le peuple athénien puisse comprendre comment le personnage foudroyé avait pu malgré tout accéder à cette apothéose. Ainsi, la dernière partie de la tragédie doit retracer le chemin de la déchéance à la gloire, elle est perçue comme le moment où Ajax pouvait se transformer en objet de culte et passer de la damnation à la sanctification ${ }^{8}$.

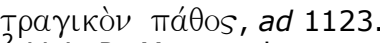

2 Voir P. Mazon, dans sa Notice à l'édition des Belles Lettres, Paris, 1958, p. 4, ou encore J. de Romilly, dans son édition commentée de l'Ajax (Paris, 1976, p. 19) : « ... on aurait pu s'attendre ... que la découverte du corps et le deuil soient la conclusion de la pièce : or, il s'en faut de près de cinq cents vers. Et c'est là un trait qui surprend et déçoit le lecteur moderne ».

${ }^{3}$ Voir Romilly, op. cit., p. 20

${ }^{4}$ Cf. Mazon, op. cit., p. 5 : « II <le lecteur moderne> a peine à accorder à la question des funérailles l'intérêt qu'y pouvait trouver un public antique »; voir encore J. C. Kamerbeek : « ...the altercations between Menelaus and Teucer and the discourses of Agamemnon and Teucer follow too much the taste of the Athenian public for lawsuit speeches, bearing in too high a degree the mark of their times » (The Plays of Sophocles, Part I, The Ajax, Leyde, 1963, p. 15). Il est vrai que R. C. Jebb oppose à ce type de critiques l'expérience du spectateur enthousiaste qu'il a été lors d'une représentation de la pièce à Cambridge en 1882 (Sophocles, VII, The Ajax, Cambridge, 1896, p. xIv, n. 1).

${ }^{5}$ Op. cit., p. xliv.

${ }^{6}$ Comme le signalent K. Reinhardt, Sophocle, trad. fr. d'E. Martineau, Paris, 1971, op. cit., p. 59, ou Kamerbeek, op. cit., p. 15.

${ }^{7}$ Cf. K. Reinhardt, en particulier p. $57 \mathrm{~s}$.

${ }^{8}$ L'appréhension du personnage prend parfois une coloration chrétienne, comme chez Jebb (op. cit., p. xxxii) : «The Athenian feeling would be analogous to that of a medieval audience witnessing a drama which concerned the life of a canonized saint, in which the doubtful issue was whether the powers of evil would succeed in making him commit some sin which would doom his soul beyond the hope of pardon »; le public aurait vu avec le plus grand intérêt le processus dont le héros sortirait malgré toutes ses faiblesses « en objet qui mérite la vénération religieuse ». Sans aller aussi loin, la majorité des commentateurs posent, en s'en réjouissant, que la fin de la pièce réalise la « réhabilitation » d'Ajax (voir par exemple C. M. Bowra, Sophoclean Tragedy, Oxford, 1944, rééd. 1960 , p. 18), la reconquête de I'honneur qui rend possible l'hommage (Romilly, op. cit., p. 21 , ou Kamerbeek, op. cit., p. 14). Quand on s'en tient à une perspective résolument historique, on voit ici, plus froidement, s'accomplir le mouvement d'intégration dans la cité : « ... les 555 vers ... qui suivent le suicide d'Ajax et qui sont consacrés au destin du corps du géant - 'comment s'en débarrasser?' - ne sont aucunement un supplément postiche. C'est précisément ce débat qui va faire du héros 'homérique' un héros civique, éponyme de la première ... des dix tribus de l'Athènes 
[386] Deux éléments antithétiques permettent à Sophocle, selon les interprètes, de rendre à Ajax toute sa noblesse. L'auteur jouerait tout d'abord sur le contraste entre la grandeur de celui-ci, héroïque jusque dans sa mort, et la bassesse des Atrides. Les interprètes ne trouvent pas de mots assez durs en effet pour qualifier l'attitude et les propos des deux chefs de l'armée achéenne. L'indignité que l'on trouve dans leurs paroles, la mesquinerie qu'ils affichent en refusant à Ajax les rites funéraires, met en valeur, selon la critique, l'immensité d'un héros qui leur est infiniment supérieur, quoi qu'il ait fait, et mérite, par là même, tout notre respect ${ }^{9}$. Ainsi, bien involontairement, les Atrides, figures d'une tyrannie haïssable ${ }^{10}$, contribuent à la gloire d'Ajax.

À l'opposé, Ulysse est le plus souvent paré de toutes les vertus. La raison en est simple : il est l'auteur conscient de cette [387] reconquête et montre par ce geste, qui annule l'inimitié passée, sa magnanimité. Face à la démesure d'Ajax et à la brutalité mesquine des Atrides, on voit en lui, avec soulagement, l'incarnation de la sagesse compréhensive et humble ${ }^{11}$. Grâce à la neutralité et à I'humanité simple qu'on lui prête, Ulysse devient parfois une sorte de spectateur idéal auquel nous pouvons d'autant plus nous identifier qu'il représente la modestie qui assure le bonheur ${ }^{12}$. De fait, il apparaît chez plusieurs interprètes comme l'homme de la modernité, qui nous fait passer d'une morale archaïque à une vision plus policée - et d'une certaine manière plus légitime - du monde ${ }^{13}$.

En dernière analyse, ce qui frappe dans ces lectures est la manière dont elles figent le texte, en s'intéressant plus aux types psychologiques ou historiques qu'elles peuvent en extraire (le héros démesuré qui reste sympathique mais qui était voué à la disparition en tant que dernier vestige d'une morale archaïque,

clisthénienne, l'Aiantis, tribu qui occupait l'aile droite, lors de la bataille de Marathon ... » (P. VidalNaquet, « Ajax ou la mort du héros », Bulletin de la Classe des Lettres et des Sciences Morales et Politiques de l'Académie Royale de Belgique, $5^{\mathrm{e}}$ série, 74, 1988, p. 463-486 [p. 482]).

${ }^{9}$ On ne donnera ici que quelques exemples d'une opinion unanime : «...La glorification du héros abattu par les dieux serait insuffisante, si la sous-mesure d'êtres pusillanimes et rancuniers ne venait donner la réplique à la démesure de la grandeur humaine... Après l'ultime ampleur du héros, quelle étroitesse ! Contre le tort qui s'est attaché à la grandeur, quelle plate légitimité!», Reinhardt, op. cit., p. 59; «...these scenes of contention and invective ... heighten our sense of Ajax's grandeur by simple contrast with these little men », R. M. Torrance, «Sophocles : Some Bearings», Harvard Studies in Classical Philology, 69, 1965, p. 269-327 (p. 279); «Their meanness and lack of dignity confirm all the more strongly our sense of the grandeur d'Ajax », P. E. Easterling dans : P. E. Easterling-B.M.W. Knox (éds.), The Cambridge of Classical Literature, vol. I, Greek Literature, Cambridge, 1985, p. 302.

${ }^{10}$ La manière dont Sophocle présente les Atrides a parfois été utilisée comme un indice pour la datation de la pièce. Ainsi Kamerbeek (op. cit., p. 16 s.), après Reinhardt (op. cit., p. 36, n. 3), voit dans la querelle entre le Salaminien Teucros et le Spartiate Ménélas une preuve que la tragédie a été composée à un moment où la souveraineté d'Athènes se sentait menacée par Sparte (avant 446). Sans être aussi précis, R. P. Winnington-Ingram lit dans les propos autoritaristes des Atrides un avertissement politique de Sophocle, qui voudrait mettre ses concitoyens en garde contre les survivances de motifs tyranniques dans le monde contemporain (Sophocles. An Interpretation, Cambridge, 1980, p. 70 s.).

${ }^{11}$ Cf. Torrance : «Such wisdom Odysseus alone, in his play, possesses », loc. cit., p. 279; ou Ph. Holt : «Odysseus' humility, moderation, and reason come as a refreshing relief to the wrangling of them all... » («The Debate-Scenes in the Ajax », American Journal of Philology, 102, 1981, p. 275288 [p. 288]).

12 «It is he, and not the Chorus, who provides the role of impartial critic on what happens ... $<$ he is $>$ presented as a faultless character ... it is his decency and his wisdom which win our support ... In Ajax we see pride and its result, in Odysseus modesty and the good ends to which it leads», Bowra, op. cit., p. 37.

${ }^{13}$ W. B. Stanford semble tenté d'en faire le porte-parole de Sophocle : « ...Odysseus's tolerant, level-headed, sane self-restraint is closer to his <Sophocle> own view of life than Ajax's pessimism» (The Ulysses Theme. A Study in the Adaptability of a Traditional Hero, 2nde éd. rev., Oxford, 1968, p. 107). H. D. F. Kitto en fait un personnage sur lequel nous pourrions encore prendre modèle en ce qu'il comprend les règles de notre existence (« ... if... we substitute the modem phrase 'the laws of humanity' we do no violence to the sense », Form and Meaning in Drama. A Study of Six Greek Plays and of Hamlet, Londres, 1956, p. 198). Cf. aussi Winnington-Ingram (op. cit., p. 71), qui voit évoqué avec Ulysse - l'homme de la sôphrosunè et de la pitié - un monde social et politique idéal, libéré à la fois de l'héroïsme et du despotisme, tous deux auto-destructeurs. 
les tyrans à courte vue dont il faut toujours se méfier, le héros véritablement humain qui mérite au plus haut degré notre estime). Les réserves qu'émet quelqu'un comme Reinhardt, qui déplore la rigidité de la discussion, trahissent clairement l'absence d'attention prêtée au déroulement des échanges. Plutôt que de s'interroger en effet sur le sens que peuvent prendre l'utilisation de l'agôn de la comédie et l'impossibilité de dialogue que mettent effectivement en scène les discussions qui opposent Teucros à Ménélas, puis à Agamemnon, avant le retournement opéré par Ulysse, on s'en tient à un jugement de valeur extérieur au drame qui se déroule : la discussion reste formelle, elle n'aboutit pas, ceci est donc un signe supplémentaire de l'archaïsme de la pièce ${ }^{14}$.

[388] Par ailleurs, derrière l'apparente harmonie que semblent globalement présenter les lectures proposées se dissimulent des disparités qui révèlent en réalité les problèmes mêmes que posent ces interprétations. Kitto s'élève à juste titre contre la confusion souvent faite entre religion cultuelle et drame religieux et objecte à ceux qui voient s'accomplir ici la réhabilitation d'Ajax que la tragédie ne met à aucun moment en scène son héroïsation. Son interprétation n'en demeure pas moins elle-même très générale et morale, et fait autant abstraction que les autres des différents échanges, puisque Kitto substitue à l'intérêt pour le culte d'Ajax le problème, plus universel, du sens religieux à donner au rite funéraire. La discussion sur l'inhumation vise, selon lui, à montrer que cet acte est la situation humaine ultime que les hommes doivent respecter s'ils ne veulent pas perdre eux-mêmes leur humanité ${ }^{15}$.

D'autres ont, sinon contesté, au moins relativisé la réhabilitation d'Ajax, montrant ainsi que le problème était peut-être plus complexe qu'il n'apparaissait. Là où une majorité d'interprètes ont finalement vu un dénouement heureux, quelques-uns ont repéré certaines failles. Il faut citer ici en particulier B. M. W. $\mathrm{Knox}^{16}$ et Ch. Segal ${ }^{15}$. Le premier rappelle ce qui pourrait sembler une évidence, mais qui est pourtant très rarement exprimé : Ajax, l'homme qui a fait le choix de la permanence, qui est mort pour ne pas renoncer à sa haine contre ses ennemis, « aurait préféré mourir plutôt que de reconnaître en Ulysse un ami $\gg^{18}$. Toutefois Knox, qui s'attache finalement, lui aussi, davantage à définir des attitudes éthiques qu'à analyser le déroulement de la pièce, ne s'attarde pas à la portée de cette remarque. Il met pourtant le doigt sur un élément essentiel de la pièce. Certes, du point de vue de I'Histoire, auquel se limite en fait l'ensemble de la critique, l'ordre est rétabli, voire établi, à la fin de la pièce ; cependant, du point de vue d'Ajax, qui doit compter avant tout autre pour comprendre la pièce, puisque avant d'être un héros de culte, il est, même mort, le héros de la tragédie qui porte son nom, il ne peut être qu'inconcevable d'obtenir sa part de gloire d'Ulysse, I'homme qui est à l'origine de la catastrophe en ayant gagné les armes d'Achille.

[389] Segal, quant à lui, repère le caractère problématique et contrasté de la gloire dévolue à Ajax à la fin de la pièce. Selon lui, Sophocle «juxtapose de manière paradoxale la monumentalité et la précarité $\gg^{19}$, en situant la « renommée impérissable » dans l'édification hâtive d'un tombeau, en le vouant à l'éternité grâce à une tombe dont l'humidité évoque la corruption (cf. $\epsilon \hat{v} p \omega ́ \in \mathcal{L} \in \alpha$, v. 1167$)^{20}$. Il avait aussi remarqué plus tôt, selon le même système de polarité qui lui est cher, que l'espace sauvage où est tombé Ajax devient un lieu de civilisation, en devenant celui de sa réintégration sociale ${ }^{21}$. Segal inscrit ces antinomies dans une réflexion sur l'essence du drame et insiste donc surtout sur

\footnotetext{
${ }^{14}$ Cf. Reinhardt, op. cit., en particulier p. 59 s.

${ }^{15}$ Op. cit., p. 182 s. et passim.

16 «The Ajax of Sophocles », Harvard Studies in Classical Philology, 65, 1961, p. 3-37.

17 Tragedy and Civilisation. An Interpretation of Sophocles, Cambridge (Mass.)-Londres, 1981, p. 109151; Sophocles' Tragic World. Divinity, Nature, Society, Cambridge (Mass.)-Londres, 1995, p. 16-25.

${ }^{18}$ Cf. p. 28.

${ }^{19}$ Segal (1995), op. cit., p. 25.

${ }^{20}$ Cf. p. 23 et 25.

${ }^{21}$ Segal (1981), op. cit., p. 127.
} 
la juxtaposition de deux dimensions temporelles, épique et dramatique. On peut pourtant ici encore resserrer la perspective et s'interroger sur le sens que prennent ces contrastes dans la pièce. Il y a bien quelque chose de paradoxal dans cette gloire accordée à Ajax, dans ce lieu retiré, par son pire ennemi. Ces anomalies doivent avoir pour but de montrer que le kleos ici ne va pas de soi, ce d'autant moins qu'il n'est nullement le résultat d'un consensus. Les interprètes, peut-être trop enthousiastes pour s'arrêter à une constatation qu'ils font cependant tous, n'insistent pas sur le refus catégorique qu'opposent les Atrides à l'éventualité de l'ensevelissement. En fait, Ulysse se retrouve seul maître de la décision.

L'engouement de la critique pour celui-ci l'a amenée, sur ce point encore, à simplifier les choses. Soit on ne se pose même pas la question de savoir pourquoi c'est précisément à Ulysse, l'ennemi d'Ajax, et, traditionnellement, I'homme des mots et de la ruse, que Sophocle donne ce rôle si important que certains interprètes tendent à faire de lui le héros principal ${ }^{22}$. Soit on se voit contraint, pour défendre dans l'Ajax la figure d'un Ulysse humaniste, de distinguer deux Ulysse, I'un noble, l'autre plus vil, deux périodes dans la présentation du personnage par Sophocle, qui idéaliserait le héros dans I'Ajax, et le réduirait à sa réputation d'homme roué et prêt à tout dans le Philoctète, même deux moments dans l'Ajax : le début de la pièce, qui nous montrerait I'Ulysse nocturne et rusé de la Dolonie, et la transfiguration finale ${ }^{23}$. Une analyse plus précise de l'argumentation du personnage permettrait, cette fois encore, de résoudre le problème.

[390] Ainsi, il paraît nécessaire de relire attentivement cette dernière partie de la pièce. On peut bien sûr affirmer que s'y trace le chemin de la chute à la gloire, mais en restreignant l'intérêt au problème de I'héroïsation athénienne d'Ajax, on perd de vue la question essentielle que pose l'ensemble de la pièce et qui est soulevée, sur un autre mode, ici : celle du kleos. Ajax, pourtant si sûr de l'obtenir ${ }^{24}$, est mort à cause de lui, parce qu'il ne pourrait pas, comme son père, revenir chez lui paré de la « bonne renommée » ( $\epsilon \hat{\kappa} \kappa \lambda \in \iota \alpha, v .436$ et 465). À travers le problème de l'ensevelissement, c'est bien évidemment celui de cette gloire qui se pose encore. Les Atrides la lui refusent. Mais, ce faisant, comme on l'a d'ailleurs parfois entrevu ${ }^{25}$, ils vont se perdre eux-mêmes. Ils vont en effet se montrer incapables d'invoquer un critère qui puisse véritablement imposer l'autorité dont ils se réclament pour faire rentrer dans le rang l'individu Ajax et interdire d'ensevelir l'encombrant cadavre.

Teucros, dont on a souvent mal compris l'action, va contribuer à mettre en pleine lumière cette impuissance. Contrairement à ce que l'on dit en général, les deux frères ne sont pas d'emblée caractérisés par leur bassesse, mais la polémique violente qui s'engage avec Teucros va permettre de révéler la faiblesse de leur position face à une question réelle, et difficile, que ni l'un ni l'autre ne parviennent à résoudre : comment rendre compte de l'individu,

\footnotetext{
${ }^{22}$ Voir Kitto ou Bowra.

23 Stanford (op. cit.) distingue deux aspects, tout aussi traditionnels l'un que l'autre, d'Ulysse : l'«Autolycien » et l'homérique, dont la magnanimité, sans être dénuée de toute ambiguïté, I'emporterait toutefois selon lui. L'Ajax reprendrait le portrait homérique, tandis que le Philoctète, où la vilenie et le cynisme d'Ulysse ne font pas l'ombre d'un doute pour Stanford, exploiterait la face négative du héros; le début de la pièce, où le personnage semble « moins honorable », serait une concession au préjugé populaire anti-ulysséen ou bien un exemple supplémentaire du caractère plus souple de l'héroïsme que lui ont accordé les poèmes homériques (cf. p. 104-108). Voir encore J. Boulogne, qui ne nuance pas autant que le fait Stanford la présentation d'Ulysse dans les poèmes homériques et considère que Sophocle se démarque de la tradition dans l'Ajax en y idéalisant Ulysse alors qu'il reprendra dans l'autre tragédie «l'image unique d'un rusé » que nous offrent l'Iliade et l'Odyssée («Ulysse : deux figures de la démocratie chez Sophocle », Revue de Philologie, 62, 1988, p. 99-107).

${ }^{24}$ Voir le vers 769.

${ }^{25}$ Voir Knox, op. cit., p. 28.
} 
qu'Ajax, cet énorme moi, incarne de manière hyperbolique, dans le cadre d'une communauté soumise à des règles qui ne peuvent le nier. Leurs arguments se montreront totalement inadéquats. La décision de Ménélas repose sur une conception tyrannique du pouvoir, fondée sur la peur. Mais comment intimider un héros comme Ajax, dont la fonction protectrice au sein de l'armée exige qu'il soit exempt de toute crainte ? L'aporie réduira Ménélas au silence. À l'opposé, Agamemnon, qui semble plus démocrate, parle au nom de la justice, mais on verra que sa conception du droit ne parvient pas, elle non plus, à résoudre [391] le problème posé par Ajax. Les deux formes traditionnelles d'autorité que l'un et l'autre invoquent se révèlent totalement inopérantes, et l'on est donc loin d'une situation d'apaisement : Ajax, par l'intermédiaire de Teucros, et d'Ulysse tout autant, va entraîner dans sa chute les chefs de l'armée achéenne.

On ne rejoint pas ainsi l'opinion selon laquelle Sophocle ferait table rase d'une morale archaïque de type homérique pour promouvoir des idées plus adaptées à l'évolution des temps. L'Histoire a peut-être donné raison à Ulysse, mais ce qui importe est de comprendre le rôle qu'il joue à l'intérieur même de la tragédie. Certes, le discours d'Ulysse, contrairement à celui de Ménélas et d'Agamemnon, parvient à rendre compte de la dimension héroïque d'Ajax, à qui il octroie son kleos. Le fait même de pouvoir dire les choses de la sorte signale toutefois le problème : non seulement l'objet tant désiré est offert par le pire ennemi, mais il est le produit d'un discours, et non pas directement, comme aurait pu l'espérer Ajax, d'actes glorieux. De plus, ce discours, que l'on pourrait qualifier de sophistique tant il relativise le réel, est, comme tel, assumé par un individu et non par la collectivité : c'est donc la notion même de kleos qui est dès lors remise en question et prend une tournure dont Ajax n'aurait pu rêver dans ses pires cauchemars. Ainsi, comme je vais essayer de le montrer, le dénouement ne coïncide pas avec une véritable résolution, car la question de fond reste, malgré les apparences, ouverte. Par là même, la violence extrême de la première partie de la pièce ne fait pas place à l'espoir, mais un tragique succède à un autre : au tragique de l'action vient maintenant s'ajouter celui qui réside dans la manière dont cette action elle-même, le sens qu'elle voulait se donner, sont niés par la nature du kleos concédé à Ajax.

Le débat qui va s'engager autour du corps d'Ajax a, tout au moins pour les engagements qui opposent Teucros aux Atrides, surpris par sa violence. On s'étonne de l'« étroitesse d'esprit», de la «vulgarité » dont font preuve les Atrides. Teucros, malgré toute la sympathie qu'il inspire en tant que courageux défenseur d'Ajax, n'est pas épargné lui non plus, dans la mesure où I'on déplore qu'il accepte de leur répondre sur le même ton sans tenter d'élever le débat ${ }^{26}$. Toutefois, la réaction immédiate que provoque la brutalité des échanges ne doit pas occulter un [392] problème bien plus grave qu'une défaillance de caractère : c'est la légitimité même de ceux qui ont statué sur le sort d'Ajax qui est mise en danger.

La manière tout d'abord dont s'agence la parole dans le début de l'échange entre Ménélas et Teucros est signifiante. Malgré une entrée en scène qui se veut autoritaire, puisqu'il défend d'emblée à Teucros (en 1047 s.) de toucher au corps d'Ajax, Ménélas est sommé de s'expliquer. La question de la raison qui justifie une telle injonction est redoublée. Teucros tout d'abord met en doute que Ménélas puisse parler de sa propre autorité. En examinant la réponse que donne celui-ci au vers 1050, on peut en effet être tenté de considérer Tívos, en 1049, à la suite de Kamerbeek, non pas comme un neutre, ainsi que les commentateurs le font le plus souvent, mais comme un masculin : «pour qui astu prodigué des paroles aussi énormes $\gg^{27}$. Le $\phi \omega \nu \omega \tilde{\omega}$ du vers 1047 est corrigé :

\footnotetext{
${ }^{26}$ Cf., entre autres, Torrance, p. 279, Winnington-Ingram, p. 62. Jebb résume assez bien un sentiment largement répandu lorsqu'il dit que la scène entre Teucros et Ménélas est « parfaitement répugnante pour le goût moderne », p. xliv.

27 L'intérêt d'une telle lecture réside aussi dans le fait que la question du motif n'est pas ainsi simplement répétée d'un vers à l'autre.
} 
Ménélas doit renoncer à l'expression d'une décision propre. Il affirme encore son

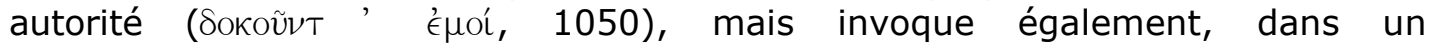
renchérissement, celle de son frère, présenté, pour que l'arrêt ne soit plus contestable, dans son rôle de chef de l'armée (« ce sont des paroles qui m'agréent, et qui agréent à celui qui commande l'armée ${ }^{2828}$ ). Teucros, visiblement peu impressionné, revient alors à la charge, en interrogeant son interlocuteur sur la raison d'une telle décision. Ménélas, même s'il s'exprime le premier, est placé dans la position de l'accusé : les rôles sont renversés.

Le discours qui suit, dont on a unanimement reconnu la violence, est lui aussi fragile à plus d'un titre. Au départ, il n'a pourtant rien de scandaleux. Le motif du refus d'ensevelir Ajax est présenté de manière objective. Ménélas ne fait que redire ce que l'action depuis le début de la tragédie nous a montré : I'intention meurtrière d'Ajax, l'intervention de la divinité, le bras du héros détourné sur le bétail pour que soient préservés les Achéens (voir les vers 1052-1065). La suite toutefois montre que la situation n'est en fait pas aussi simple.

Les vers 1067-1069 semblent particulièrement odieux. Ménélas, conseillant à Teucros de ne rien tenter de violent (1066), s'explique : « car si nous n'avons pu nous en rendre maîtres quand il [393] <Ajax> vivait, nous en serons les chefs absolus une fois mort, même si tu n'y consens pas, et la correction ( $\pi \alpha \rho \in v \theta u ́ v 0 \nu T \epsilon S, 1069)$ se fera par nos bras ». Ce qui peut apparaître comme de la lâcheté est surtout l'aveu d'une totale impuissance, qu'illustre bien le choix de

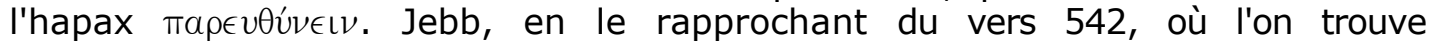
l'expression $\chi \in \rho \sigma i \nu \in \hat{U} \theta \hat{v} \nu \omega \nu$, à propos d'Eurysacès, comprise en général comme l'action de guider les pas mal assurés de l'enfant, pense que le groupe de mots signifie « en le guidant de nos mains ». Comme le remarque Kamerbeek, il ne tient pas compte ainsi du préverbe $\pi \alpha \rho \alpha-$. Deux sens ont été donnés à celui-ci. Campbell, qui traduit par « en le maintenant dans le rang ${ }^{29}$, voit suggérée ici l'image d'un garde-chiourme qui marche à côté des esclaves pour les maintenir en ligne. Kamerbeek, lui, préfère, avec une scolie tardive, donner au préverbe le

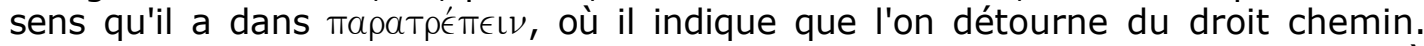
Il ne va pourtant pas au bout du raisonnement, et commente en recourant à l'image du cheval rétif qui ne supporte pas d'être guidé.

Il faut en fait davantage exploiter la contradiction qu'il y a dans le terme même. Eủ日úvelv signifie « redresser ». C'est le verbe que l'on trouve chez Solon (4W., v. 36) quand il évoque l'Eunomie qui « redresse les sentences torses ${ }^{30}$. Ménélas aurait pu ainsi tout simplement dire qu'il s'agit de remettre Ajax dans le droit chemin. L'utilisation du préverbe, avec le sens qu'il prend dans un verbe comme tapaßaı́v $\in \nu$, donne toutefois à la proposition un caractère paradoxal ${ }^{31}$. La ligne droite constitue une déviation, une violence donc, confirmée par l'emploi de $\chi \in \rho \sigma i ́ v$, qui s'oppose à la force de persuasion dite dans le vers suivant (1070, « il n'a absolument jamais consenti de son vivant à entendre mes paroles »). Le verbe dit l'existence de deux trajectoires, tout aussi contraignantes l'une que l'autre, et donc absolument incompatibles. Comme le reste de la pièce l'a montré, en particulier les propos rapportés par le messager aux vers 767-775, Ajax suit sa ligne propre, qui ne tient pas compte des dieux, et qui doit ignorer, a fortiori, les injonctions des hommes.

Ainsi, même si l'on peut traduire ses propos en termes d'une lâcheté empreinte de dépit, Ménélas ne fait que constater une évidence. La mort d'Ajax

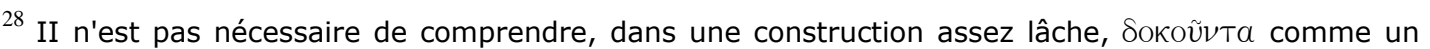
neutre pluriel (voir Jebb; Kamerbeek ou Stanford sont plus hésitants). Comme souvent dans les stichomythies, un mot de la phrase précédente est repris implicitement et se voit attribuer un autre qualificatif : le participe est sur le même plan que Toбó $\delta € \epsilon$ en 1049, qu'il corrige.

${ }^{29}$ « Keeping him in order», Sophocles, vol. II, Oxford, 1881, ad loc.

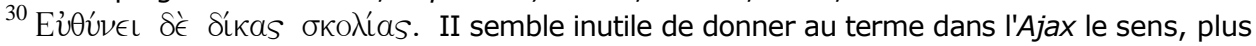
métaphorique, de « punir » (que l'on retrouve d'ailleurs dans notre français « corriger »), comme dans Platon, Protagoras, 326e.

${ }^{31}$ Bailly a ressenti le paradoxe en traduisant le verbe par « diriger hors du droit chemin », qu'il glose par « diriger à son gré », introduisant ainsi l'idée d'arbitraire.
} 
est la condition sine qua non de sa [394] soumission. Le héros, tout comme Achille, sur lequel Agamemnon dans I'Iliade n'a qu'une emprise toute relative, est une individualité qui, contrairement au chef de l'armée achéenne, s'est imposée aux autres par sa nature même. Il en résulte une tension constante entre l'expression de cette singularité et l'autorité sous laquelle il doit en principe combattre. Et il est en réalité nécessaire qu'il en soit ainsi. La comparaison avec l'âne qui est utilisée à son propos dans I'Iliade (XI, 558-571) est de ce point de vue significative : comme l'âne obstiné ne bouge pas malgré les coups de bâton, Ajax se maintient seul face à l'ennemi. Il serait faux de dire qu'il ne connaît pas la peur, mais le poème précise à chaque fois que la frayeur, quand il la subit, lui est inspirée par Zeus (XI, 544, et XVI, 119) ${ }^{32}$. Comme le prouvent les épisodes qui illustrent son action intrépide, et solitaire, de défenseur des nefs, les guerriers les plus offensifs ne provoquent en lui aucune frayeur. Sa fonction même de défenseur explique cette inflexibilité : par essence, Ajax ne doit pas craindre les hommes ${ }^{33}$.

Cette assurance a son revers; Agamemnon et Ménélas ont beau être les chefs de l'armée, ils n'en sont pas moins des hommes qui risquent, à ce titre, de se heurter eux aussi à la forme d'héroïsme qu'incarne Ajax. Le début du drame l'a bien montré : si Ajax, dont l'Iliade dit qu'il est le meilleur des Achéens après Achille, s'en prend aux Atrides et conteste le jugement des armes qui a donné l'avantage à Ulysse, c'est parce qu'il considère qu'ils nient l'indéniable, qu'Ulysse lui-même admettra ( $v .1340$ s.), à savoir sa prééminence dans l'armée après la mort d'Achille (cf. les vers 442-444). Il est paradoxal d'être à la fois le « meilleur des Achéens » et de devoir se soumettre à l'autorité d'hommes nécessairement moins valeureux, et c'est à mon sens ce problème insoluble que souligne la tragédie.

Cette difficulté, inhérente au monde héroïque, mais qui demeure effective bien au-delà, explique la faiblesse de la suite du discours que tient Ménélas (10711086). Tous les commentateurs ont remarqué que la notion-clé y était la peur :

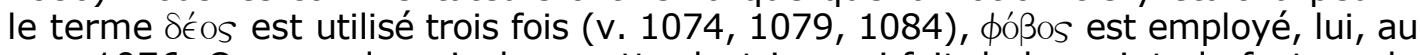
vers 1076. On a voulu voir dans cette doctrine qui fait de la crainte le facteur du bon fonctionnement de la cité ou de l'armée une allusion à la pratique Spartiate du pouvoir ${ }^{34}$. On a aussi [395] repéré un certain flottement dans les propos de Ménélas, qui semble confondre, ou assimiler, de manière injustifiée, le pouvoir à exercer dans la cité $\left(1073 \mathrm{~s}\right.$.) et dans l'armée $\left(1075 \mathrm{~s}\right.$.) ${ }^{35}$. En réalité, Ménélas ne confond pas tant les domaines qu'il ne les accumule, du plus général au plus particulier, comme autant de preuves de la justesse de ses dires : la cité avec ses lois, l'armée et l'individu (cf. 1077-1080), bref toutes les composantes d'un

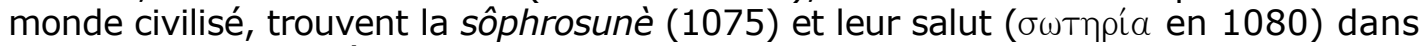
la peur. Le plus intéressant ici n'est pas de savoir si le roi de Sparte illustre une théorie politique, historique, mais de comprendre ce que vise une généralisation aussi appuyée. Ménélas assigne à ses propos une portée d'autant plus vaste que le discours qu'il tient n'a absolument aucune pertinence dans le cas d'Ajax,

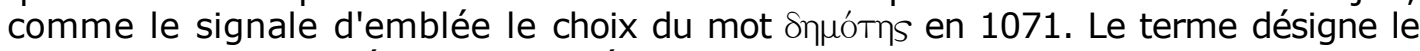
petit peuple de la cité ou de l'armée, un Thersite par exemple, mais ne peut en

\footnotetext{
${ }^{32}$ La peur se manifeste à chaque fois dans la même circonstance : celui d'un affrontement direct avec Hector, et elle est intégrée au chant XVI dans le plan de Zeus qui «voulait la victoire pour les Troyens» (v. 121).

${ }^{33}$ Ajax, dans la pièce, intègre lui-même cette absence de peur dans la définition de son être : cf. les vers $545-7$.

${ }^{34}$ Cf. Jebb, ad 1076, ou Bowra, op. cit., p. 52; voir aussi ci-dessus, n. 10.

35 Ch. Elliot Sorum, constatant que Ménélas, tout comme Agamemnon par la suite, selon elle, applique les règles de la discipline militaire à l'Etat, l'armée et l'individu, conclut à une mauvaise perception par les Atrides de leur position, dans la mesure où une telle politique peut s'appliquer à une situation où l'armée et la cité ne font qu'un, mais sûrement pas au contexte épique («Sophocles' Ajax in context», Classical World, 79, 1986, p. 361-377; cf. p. 374 s.). Elle perçoit là, avec raison, l'un des indices du caractère intenable du code héroïque, mais l'inclut dans une interprétation générale qui fait de la pièce une réflexion, historique, sur le conflit qui oppose l'idéal traditionnel, individualiste, à la réalité, de type hoplitique, du Ve siècle.
} 
aucun cas s'appliquer à Ajax, qui, de toute façon, par nature, est étranger à la peur : l'Atride est obligé de «rétrécir» Ajax, de l'assimiler au vulgaire, pour le faire entrer dans un cadre qu'il puisse maîtriser. Le risque est grand. Pour défendre la possibilité même d'existence d'une autorité dans ce monde de héros, Ménélas met en péril la crédibilité de son propos, et, au-delà, précisément, de sa fonction de chef. En d'autres termes, en voulant justifier une position nécessairement intenable, puisqu'elle est obligée de nier tout le passé glorieux du héros pour châtier le délit effectif du soldat, membre d'une communauté militaire, Ménélas est condamné à un discours inadéquat.

L'Atride finit par s'enliser dans la contradiction. Après avoir en effet donné à Teucros une leçon d'alternance, en lui expliquant que l'hybris dont est coupable $\mathrm{Ajax}^{36}$ se paie nécessairement (cf. v. 1081-1086), Ménélas semble vouloir démontrer la véracité de ses dires en faisant de l'événement présent la preuve de l'accomplissement effectif de cette loi. « Les choses viennent à leur tour. Avant, cet homme était bouillant et insolent (íßploTńs), [396] c'est à moi

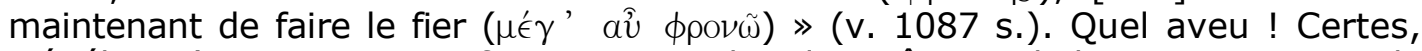
Ménélas n'est pas assez fou pour se dire lui même « hybristique », mais le

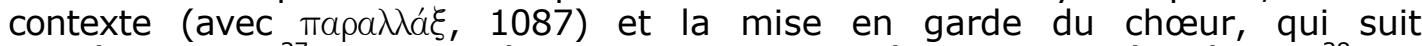

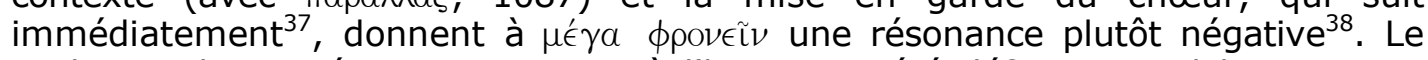
seul type de pensée qui convienne à l'homme a été défini par Calchas au vers

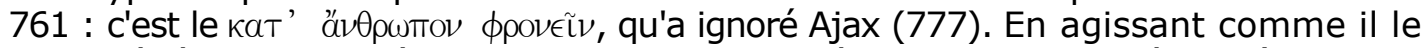
fait, Ménélas reconnaît implicitement qu'il opère selon le modèle même qu'il vient de rejeter.

Teucros relèvera plus tard le caractère « hybristique » de Ménélas (cf. 1151). Pour l'instant, il s'empresse de souligner le manque de pertinence du discours politique et moral qu'il vient de subir, en présentant comme une usurpation de droit la généralisation opérée par Ménélas. Il nie d'abord implicitement la possibilité de faire d'Ajax un dèmotès en rappelant qu'il était son propre maître dans cette expédition (1099). Il signale ensuite à Ménélas que son pouvoir sur Sparte ne peut nullement s'étendre aux Salaminiens (1102 s.), puis lui assène qu'il n'a aucun pouvoir sur l'ensemble de l'armée, mais qu'il est lui-même subordonné à un autre (1105 s.). Il lui refuse enfin le statut d'interlocuteur valable aux vers 1114-1117, excessivement méprisants, ce que lui fera payer Agamemnon en le traitant de la même manière.

Ménélas a la faiblesse de poursuivre une discussion dont il va sortir anéanti. Il serait trop long d'examiner le détail de l'échange, mais il vaut la peine de mettre en évidence certains éléments de l'action corrosive du demi-frère d'Ajax. Ménélas commet d'emblée l'erreur de se placer sur le terrain où l'a entraîné Teucros : celui de la valeur, en tentant à son tour le mépris $(1120)^{39}$. L'autre ne se laisse pas impressionner mais surenchérit dans l'insulte (1123) et va ensuite s'imposer dans la discussion, où il s'ingénie à démonter le discours de son adversaire. C'est en fait Teucros qui mène le jeu. Tout d'abord, il tourne en ridicule la première exagération de Ménélas, en $1126 \mathrm{~s}^{40}$. Il amène ensuite un autre [397] élément que Ménélas a totalement ignoré jusqu'à présent dans son discours, alors qu'il est important et joue un rôle essentiel dans la pièce : le rapport aux dieux. Ménélas n'a fait qu'évoquer jusqu'alors l'aide que lui a apportée la

\footnotetext{
${ }^{36}$ Le terme est employé à propos de son projet meurtrier en 1061.

37 « Ménélas, après avoir posé des sentences d'expert, ne fais pas toi-même ensuite l'insolent envers

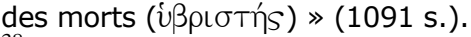

38 Voir D. L. Cairns, « Hybris, Dishonour, and Thinking big », Journal of Hellenistic Studies, 116, 1996, p. 1-32 (cf. p. 11 s.), pour une opinion analogue.

${ }^{39}$ II n'est pas nécessaire de penser que le mépris pour l'archer fait référence à l'époque classique, où les archers sont des barbares et l'arc l'arme des Perses et de la police athénienne, composée en grande partie de Scythes (voir Jebb, par exemple). La dévalorisation peut se trouver dans I'Iliade, où l'archer est du côté de la trahison, avec Pandare, au début du chant IV, qui rompt le pacte, et Pâris, en XI, qui blesse Diomède. Mais Teucros a beau jeu de répliquer, en songeant sûrement à d'autres archers, bien plus valeureux, et grecs ceux-là : Philoctète et, bien sûr, Héraclès.

${ }^{40} \mathrm{M}$. « Est-il juste que cet homme triomphe alors qu'il m'a tué ? », T. « Tué ? voilà un mot singulier, si vraiment tu vis tout en étant mort ».
} 
divinité (1128 rappelle 1057); Teucros lui rappelle désormais qu'il existe aussi des devoirs à respecter si l'on ne veut pas être impie. Puis il contraint Ménélas à reconnaître une autre exagération quand il le force à préciser ce qu'il entend par

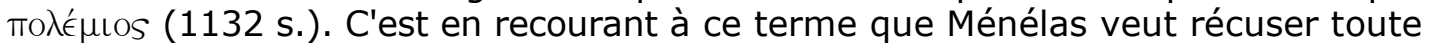
accusation d'impiété : « <j'interdis> d'enterrer mes propres ennemis. Ce n'est pas convenable en effet». Le mot désigne, comme l'a remarqué Jebb, un ennemi public. Subtilement, Teucros, s'appuyant sans doute sur l'emploi de aủròs aủtoũ en 1132, force son adversaire à réfléchir à la pertinence du terme pour Ajax, amenant ainsi Ménélas à avouer que l'inimitié se réduit ici à une haine personnelle ${ }^{41}$, ce qui invalide aussitôt son argument.

Teucros attaque enfin Ménélas sur un autre terrain important que l'Atride n'a pas occupé, comme il l'aurait dû en tant que gouvernant ${ }^{42}$, celui de la justice. D'emblée, le demi-frère d'Ajax s'est mis de ce côté (1125) en en faisant

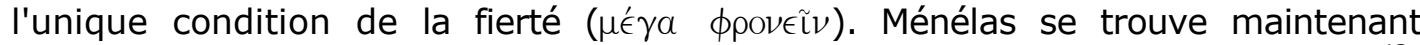
clairement en position d'accusé (1135-1137). Accusé de fraude dans le jugement ${ }^{43}$, rejetant toute responsabilité sur les juges, et acculé, pour toute réponse, à la menace, Ménélas perd définitivement le statut de chef qu'il avait voulu imposer au début de l'échange.

Les deux petits discours qui suivent (1142-1158) entérinent la défaite de l'Atride et la rupture du dialogue. Ménélas recourt à la forme traditionnelle de la fable (I'ainos), mais la détourne de sa visée habituelle. L'apologue ne finit pas, comme il le devrait, sur une parénèse, mais la leçon se réduit, cette fois encore, à la menace, dernier recours de qui ne peut plus convaincre. Teucros, lui, parodie explicitement Ménélas ${ }^{44}$. La nature corrosive de la tirade ne se réduit pas cependant au ton extrêmement ironique qu'il adopte. Ménélas est clairement accusé d'hybris (1151) et son [398] interlocuteur retourne contre lui l'accusation qu'il avait prononcée contre Ajax. De plus, Teucros se pose de la sorte en moralisateur, volant ainsi à Ménélas le rôle qu'il voulait, et n'a pas su, jouer en usant de la fable. La parénèse qui devait en principe être l'aboutissement de l'ainos se profile en effet dans ce qui se donne clairement comme un simulacre de fable ${ }^{45}$. Le travail de démolition entrepris par Teucros est achevé dans cette nouvelle inversion des rôles : c'est l'accusé initial qui sermonne celui qui se glorifiait de son statut de maître, et l'emploi de l'adjectif ăvoßßos en 1156, clairement adressé à Ménélas ${ }^{46}$, n'est pas fortuit. L'ô’ßos est traditionnellement l'apanage des rois, l'adjectif prive donc Ménélas de toute prétention à ce titre.

Teucros, en mettant en évidence des failles et des manques que trahissait déjà le discours de Ménélas, fait de celui-ci le grand perdant de l'échange ${ }^{47}$, puisqu'on assiste à l'effondrement de ses prétentions à l'autorité. Agamemnon va subir un sort analogue.

\footnotetext{
${ }^{41}$ Comme Jebb l'a remarqué (ad 1134), Ménélas devrait poursuivre ainsi : « s'il n'était pas mo $\lambda \epsilon ́ \mu$ ios

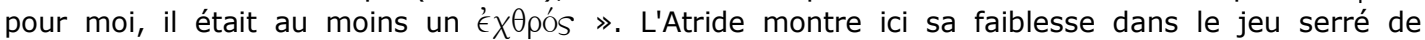
questions-réponses que lui impose Teucros. Il aurait pu en effet rétorquer qu'Ajax, en s'en prenant aux chefs de l'armée, donc à des personnages publics, se transformait en ennemi commun. Ajax d'ailleurs a maudit l'ensemble de l'armée en 844.

42 On trouve seulement le verbe $\delta$ เкaıoũv en 1072, la justice se réduisant ici, pour l'homme du peuple, à écouter ses supérieurs.

${ }^{43}$ Teucros précise ici une allusion faite par Ajax en 446.

${ }^{44}$ Voir la symétrie du vers 1150 par rapport à 1142 . Cf. aussi la question finale : « ai-je bien fait un ainos ? », 1158.

451154 s. sonnent en effet comme un commandement moral.

${ }^{46}$ Voir le vers suivant, qui explicite une identité qui était de toute façon déjà parfaitement évidente.

${ }^{47}$ Le détail en donne d'autres signes. Alors que Ménélas exprime sa menace au potentiel (1147-49), Teucros, de manière plus assurée, emploie le futur pour exprimer sa leçon comminatoire (1155). Les deux discours sont symétriques, mais celui de Teucros est plus long d'un vers. Enfin le demi-frère d'Ajax a le dernier mot, dans une tirade basée sur le renchérissement : au aíxpóv de Ménélas

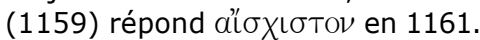


Si le ton d'Agamemnon est encore plus méprisant que celui de Ménélas, que son frère veut clairement venger ${ }^{48}$, le discours (1226-1263) semble substituer à la force le droit et la raison. Pourtant, cette fois encore, cela va se faire au prix de quelque chose d'essentiel : la négation de ce qu'a pu être Ajax - et, par conséquent de tout discours de type héroïque -, dont l'Atride lui-même ne sortira pas indemne.

Le discours d'Agamemnon se distingue de celui de son frère par une affirmation plus nette de l'importance de la justice. Si l'on compare en effet les vers 1246-1249 à 1073 s., on constate que la loi n'est plus ici liée à la peur mais à la justice. C'est un discours clairement démocratique que défend Agamemnon, qui fait prévaloir la décision de la majorité (cf. le vers 1243). Knox remarque que le chef des Achéens, comme d'ailleurs Ménélas avant lui, use de termes que I'on ne trouve pas ailleurs [399] dans Sophocle et qui « évoquent l'atmosphère $d u$ tribunal athénien $d u V^{e}$ siècle ${ }^{49}$. L'anachronisme a pour fonction, selon lui, de créer un contraste entre Ajax, le dernier des héros, représentant de l'éthique individuelle homérique, et la société de l'époque de Sophocle, dont la morale, plus communautaire, aurait fini par l'emporter ${ }^{50}$. C'est donner de la pièce une vision trop statique et historiste. Mettre dans la bouche du héros homérique des mots qui renvoient à un système politique qui lui est totalement étranger est plutôt un signal qui indique une anomalie, voire un dysfonctionnement. De fait, le discours d'Agamemnon grince.

C'est à propos du jugement prononcé sur l'attribution des armes d'Achille qu'Agamemnon proclame, au nom du respect de la loi, le caractère incontestable de la décision prise par la majorité des juges (v. 1239-1245). Toutefois, l'attribution des armes ne s'est pas faite dans le contexte d'une procédure juridique mais bien d'un concours (cf. ả $\gamma(\tilde{\nu} \nu a s$ en 1240). D'ailleurs, les termes utilisés à propos d'Ajax et des siens sont ceux que l'on emploie pour

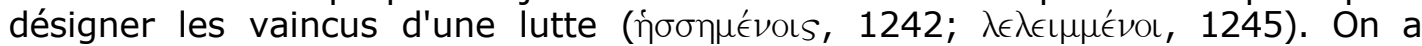

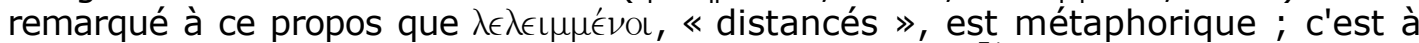
l'origine un terme utilisé dans le contexte des courses ${ }^{51}$. Or le verbe est utilisé dans ce sens plusieurs fois au chant XXIII de l'Iliade ${ }^{52}$, et le vers 1245 , par le choix du vocabulaire, rappelle un épisode bien précis de ce même chant XXIII, où il est question d'un aiguillon illégitimement utilisé dans une manœuvre caractérisée par la ruse ${ }^{53}$. Je veux bien évidemment parler de la fraude d'Antiloque dans un autre agôn célèbre : la course des chars. Dans sa tentative de dévalorisation, Agamemnon met Ajax du côté des tricheurs, mais, ce qui est plus intéressant, les mots que lui fait prononcer Sophocle l'enracinent davantage dans le contexte homérique des jeux héroïques que dans celui des affaires judiciaires.

La cohérence du discours devient alors très problématique. On se souvient en effet que la fraude d'Antiloque amène Achille à redistribuer les récompenses non pas en fonction de l'ordre d'arrivée mais selon la valeur « naturelle » de chacun $^{54}$, et que le concurrent arrivé le dernier, Eumèle, doit obtenir le deuxième [400] prix ${ }^{55}$. Ainsi, par le discours qu'il tient, Agamemnon est en totale contradiction avec la conception de l'excellence qui dicte à Achille la répartition des récompenses. La justice dont il parle ne pourrait bien

\footnotetext{
48 Les nombreux parallèles entre les propos de Teucros et ceux d'Agamemnon montrent que le chef de l'armée répond ici, en les amplifiant, aux insultes qui lui ont été rapportées par son frère.

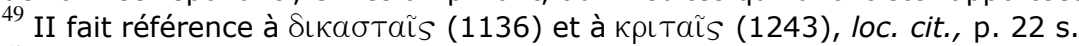

${ }^{50}$ Cf. p. 20 s.

${ }^{51}$ Voir Jebb, Kamerbeek ou Stanford.

${ }^{52}$ Aux vers 407 et 523.

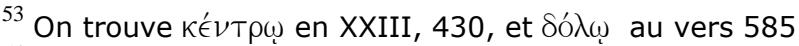

${ }^{54}$ Voir pour l'interprétation de la course de chars la thèse de Ph. Rousseau, encore inédite, $\Delta$ ı̀̀s $\delta$ '

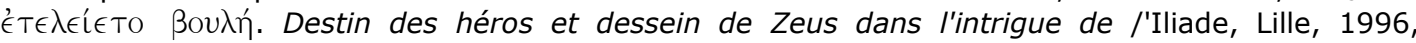
chapitre VII.

55 Cf. v. 536 ss.
} 
évidemment pas être reconnue par Ajax, et pas plus par le Ménélas de l'Iliade, qui a bénéficié du rétablissement de l'ordre d'excellence. On pourrait objecter qu'Agamemnon propose ici un autre modèle, qui repose, lui, sur le droit. Mais, ironie des mots, c'est la même $\delta$ íkn qu'invoqué le fils de Nestor au vers 542 du chant XXIII pour contester la décision d'Achille. Or Antiloque mène sa course d'une manière trop irrégulière pour qu'il puisse légitimement se placer du côté du droit.

Pour masquer cette remise en cause de la hiérarchie « naturelle » des valeurs, qui est pour Agamemnon lui-même très risquée, dans la mesure où sa fonction même, et par conséquent son importance, en dépendent, l'Atride met donc Ajax, et Teucros, du côté d'un Antiloque. La dévalorisation a commencé en fait dès les vers 1237 s., où Agamemnon met en cause l'excellence guerrière d'Ajax (« dans quel lieu a-t-il porté ses pas, à quel endroit s'est-il posté où je ne fusse pas ? Les Achéens ne comptent-ils donc pas d'autres hommes que lui ? »). Non seulement il fait preuve ainsi d'une mauvaise foi que Teucros n'aura aucun mal, ici encore, à dénoncer, mais il trahit en outre la faiblesse des critères sur lesquels reposent son autorité, car sa question de 1237 n'a pour autre effet que d'en faire un substitut possible d'Ajax, sans que soit par là affirmée sa supériorité sur le héros, bien au contraire, puisque tous ses efforts tendent à dévaloriser celuici. Ainsi, comme Ménélas, Agamemnon est contraint, pour justifier sa position, de minimiser l'action d'Ajax, en s'exposant immédiatement lui-même au travail de sape qu'il est en train d'accomplir.

Les vers 1250-1254 en donnent une illustration supplémentaire. En opposant les hommes à la pensée correcte aux êtres massifs et pourvus d'un large dos, il pense évidemment à lui-même et à $A j a x^{56}$. Il reprend une idée que l'on a trouvée dans le discours de Calchas ${ }^{57}$, mais, tout comme son frère, il la déforme en ignorant le rôle des dieux dans la hiérarchie qu'il évoque et sans voir qu'en ironisant sur le physique imposant d'Ajax, il dénigre ce qui a fait du héros le défenseur exceptionnel, et donc nécessaire, qu'il était.

[401] La comparaison avec le bœuf (1253 s.) est, elle aussi, instructive. Elle rappelle les propos de Ménélas en 1069 (voir ci-dessus). Une fois de plus, Homère est en même temps évoqué et écarté. Le bœuf frappé du fouet rappelle l'âne roué de coups de bâton, mais l'image a pour but de dire le contraire : l'animal avance ici. Surtout, Agamemnon tient de nouveau un discours à double tranchant : si Ajax est le bœuf aux flancs larges, lui-même, qui prétend le faire marcher droit, est le petit fouet. Voilà à quoi se réduit le chef de l'armée achéenne pour défendre un monde de la légitimité terriblement privé de grandeur.

Teucros, cette fois encore, va être impitoyable. Il lui est tout d'abord facile de dénoncer l'ingratitude d'Agamemnon en rappelant les exploits singuliers $\mathrm{d}^{\prime} A j a x^{58}$. Puis, pour répondre aux attaques proférées par Agamemnon contre sa naissance (aux vers 1228, 1260 s., 1263), il évoque lui-même, avec une précision inexorable, les origines de son adversaire, petit-fils de Pélops le Phrygien, d'Atrée, l'impie par excellence, de sa mère, la Crétoise aux mœurs un peu trop libres, vouée à la mort par son père (v. 1291-1297). Les héros de la guerre de Troie, déjà bien entamés, s'effondrent.

On a souvent critiqué Teucros, en jugeant que son courage et sa loyauté envers Ajax ne parviennent pas à compenser ce que l'on considère comme une «intellectual inadequacy ${ }^{59}$. Il semble en fait qu'on lui reproche de ne pas répondre à l'attente des interprètes. Ceux-ci aimeraient voir en lui un parent plein de décence et sans doute de flegme, mais se voient contraints de déplorer

\footnotetext{
${ }^{56}$ II ne retient du héros que les mensurations exceptionnelles décrites dans l'Iliade III, 227, où Ajax est dit large d'épaules et supérieur d'une tête aux Argiens.

${ }^{57}$ V. $758-761$

${ }^{58}$ Vers 1266-1287. Teucros, d'une manière sélective mais efficace, rappelle l'action exemplaire du héros lors de l'attaque des vaisseaux (voir l'Iliade, en particulier le chant XV).

${ }^{59}$ Cf. Winnington-Ingram, op. cit., p. 61 et n. 1.
} 
la « manière hystérique dont il rejette la discipline $»^{60}:$ « il est trop petit, trop rigide, trop vitriolique ${ }^{61}$. Ainsi, sa violence a gêné, parce qu'elle ne lui permettait pas de persuader les deux Atrides ${ }^{62}$. C'est une fois de plus se tromper de débat, en introduisant des aspirations d'ordre psychologique là où la tragédie nous présente des personnages qui incarnent des fonctions.

Avec la mort d'Ajax, Teucros a tout perdu : des alliés en Troade, surtout la possibilité du retour, et a fortiori de toute gloire (voir les vers 1003-1023). Sa position, comme l'illustre bien le mépris avec lequel le traitent Ménélas et Agamemnon, lui [402] interdit d'espérer convaincre les Atrides d'ensevelir le cadavre. Ce qui lui reste à faire est de démonter le discours que construisent ses adversaires pour nier la valeur d'Ajax. Son rôle, dans sa négativité même, est tragique. En exhibant les failles que les paroles des deux frères tentaient d'occulter, mais qui étaient bien réelles, il entraîne les représentants officiels de l'autorité dans la chute d'Ajax. L'aporie est à son comble.

Les interprètes, plus sensibles à l'âpreté du débat qu'à son aspect tragique, ont accueilli avec soulagement l'arrivée d'Ulysse, qui, selon eux, parvient à réconcilier ceux que Teucros, dans sa grande faiblesse, avait divisés ${ }^{63}$. La distribution même de la parole semble aller dans le sens d'une résolution. À une stichomythie entre Agamemnon et Teucros, que I'on attend pour que soit respectée la symétrie avec l'échange entre Teucros et Ménélas, se substitue un échange entre Agamemnon et Ulysse, qui semble promettre une ouverture autrement impossible. De fait, Ulysse va donner au problème sa solution à lui, et il va pour ce faire, lui aussi, mettre à mal la position de l'Atride dans une joute dont il sortira victorieux. Ainsi, sa substitution à Teucros dit moins l'apaisement qu'elle ne révèle en lui le véritable adversaire d'Agamemnon, et le gain que constitue effectivement l'ensevelissement d'Ajax ne va pas se faire sans pertes.

Le plaidoyer d'Ulysse prend le contre-pied des propos proférés par Ménélas et Agamemnon. Il se réclame d'emblée des dieux (v. 1332), qui n'ont à aucun moment fondé le discours des Atrides. La force est, quant à elle, rejetée au profit de la justice, qui devient seulement maintenant une valeur à part entière $^{64}$. De plus, contrairement à ce qu'avait fait Agamemnon, Ulysse ne généralise pas, à partir du jugement sur l'attribution des armes, le discours à tenir à propos d'Ajax, globalement dévalorisé en tant que vaincu en cette circonstance précise, mais il prend bien soin de distinguer la décision qui fait de lui, Ulysse, le détenteur des armes de l'appréciation à porter sur Ajax (v. 1336$1341)$. Le rang de deuxième après Achille que lui donne Homère dans I'Iliade ${ }^{65}$ et que revendiquait Ajax lui-même dans la tragédie lui est ici reconnu.

[403] Cela n'implique pas toutefois qu'Ulysse et Ajax aient pu se réconcilier. Le fait même qu'Ulysse puisse poser l'une à côté de l'autre, sans en problématiser le rapport, deux assertions en principe contradictoires : le rappel de sa conquête des armes d'Achille et l'affirmation de la nécessaire supériorité d'Ajax, en tant que deuxième par l'excellence, montre que les deux héros vivent dans deux univers opposés. Ajax voulait vivre dans l'ordre de la permanence ${ }^{66}$, où identité et action se confondent, tandis qu'Ulysse est l'homme du discours, et, cela va de pair, du circonstanciel. Pour régler la contradiction, on a parfois voulu croire qu'Ulysse admettait ainsi que les juges avaient pris la mauvaise

\footnotetext{
${ }^{60}$ Ibid.

${ }^{61}$ Torrance, loc. cit., p. 279.

${ }^{62}$ Cf. aussi Holt, loc. cit., p. 287.

${ }^{63}$ Voir, entre beaucoup d'autres, Holt, ibid.

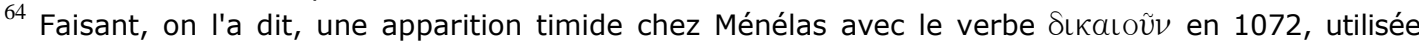
comme un instrumental par Agamemnon (1248), elle devient ici une quasi-divinité, comme le montrent l'emploi de l'article et la métaphore introduite par le verbe $\pi a \tau \in \tilde{\iota} \nu$ en 1335. Des termes

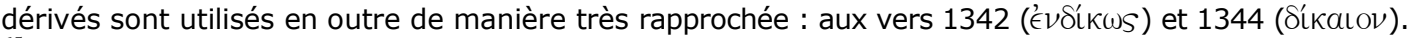

${ }^{65}$ Cf. II, 768 s., XVII, 279 s.

${ }^{66}$ Knox a raison de voir en lui le héros de la permanence et de l'absolu (loc. cit., p. 26).
} 
décision en lui attribuant les armes ${ }^{67}$. Le texte ne permet pas d'aller aussi loin. Ulysse obéit à une logique qui n'est pas celle du remords mais de l'occasion, et il en donne les clés dans deux phrases : aux vers $1344 \mathrm{~s}$. («il n'est pas juste, s'il est mort, de faire du tort à l'homme valeureux, même s'il se trouve que tu le hais »), et au vers 1347 ( « je le haïssais quand il était convenable de le haïr »). Ulysse adapte, de manière pragmatique, son action aux circonstances : il a accepté le verdict des juges, qui créait automatiquement une haine mortelle entre les deux héros ${ }^{68}$, puisque Ajax voyait nié son rang, et donc son être même, par cet usurpateur. La mort d'Ajax faisant désormais de lui sans conteste le deuxième, rien n'empêche plus Ulysse de proclamer la valeur de son rival.

Face à Agamemnon, campé dans la position du chef qui entend être obéi (v. 1352) et considère par conséquent Ajax, le rebelle, comme un ennemi, Ulysse va défendre, au-delà de l'inimitié, la mémoire du héros (1355). On a beaucoup glosé sur la grandeur dont il fait preuve dans la stichomythie ${ }^{69}$. Sans contester le succès que remporte Ulysse, je reviendrai toutefois sur un certain nombre des arguments qu'il emploie, afin de montrer en quoi sa position correspond à une conception du discours, et, au-delà, du monde, très différente de celle d'Agamemnon et d'Ajax, et, en fait, inacceptable pour eux.

[404] II faut tout d'abord insister sur le fait que le pouvoir de conviction d'Ulysse s'appuie sur la rigidité même du système de valeurs défendu par Agamemnon : ce n'est que parce qu'il est un ami que l'Atride accepte de l'entendre (cf. 1330 s.), et celui-ci ne renonce à son inflexibilité que pour prouver sa reconnaissance à cet ami, sans avoir modifié pour autant son évaluation d'Ajax (1371-1373). Rapidement, Ulysse précise le type d'ami qu'il est : il est « l'ami à la parole efficace ${ }^{70}$. De fait, il va montrer qu'il dit vrai en introduisant l'ambiguïté dans son discours et en opposant ainsi au monde dualiste de l'Atride, fait de chefs et de subordonnés, de vainqueurs et de vaincus, d'amis et d'ennemis, un monde plus complexe, grâce à une rhétorique qui relativise ce qu'Agamemnon considérait comme des valeurs absolues.

La première illustration de ce jeu sur l'équivoque apparaît au vers 1353, avec le paradoxe de la victoire qu'est une défaite infligée par des amis («Tu l'emportes quand tu es vaincu par tes amis »). Le tour est quelque peu sophistique, relativise en tout cas des notions qui étaient univoques dans la bouche d'Agamemnon. Cette acceptation conditionnelle de la défaite est reprise un peu plus loin, quand Ulysse propose une nouvelle hiérarchie des valeurs. À Agamemnon, pour qui l'inimitié est seul critère d'évaluation, sur lequel la mort elle-même n'a pas prise, son interlocuteur oppose une gradation différente (v. 1357). La complexité syntaxique de la phrase sert le propos d'Ulysse en lui donnant toute sa force. Le verbe $\nu \kappa \alpha \tilde{a}$ commande deux compléments. La proposition ne poserait pas problème avec le seul régime au génitif : «l'excellence l'emporte de beaucoup sur l'inimitié ». La difficulté réside dans l'accusatif $\mu \epsilon$. Il paraît peu justifié de faire du pronom l'équivalent d'un datif éthique $^{71}$. Pour donner à la phrase le sens attendu en rendant compte de l'accusatif : «l'excellence l'emporte sur moi beaucoup plus que l'inimitié », et en évitant de donner à moגú un sens comparatif qu'il n'a pas, Lloyd-Jones opte

\footnotetext{
${ }^{67}$ Cf. Knox, loc. cit., p. 23, ou Winnington-Ingram, p. 58 s. et n. 4.

${ }^{68}$ Cf. le vers 1336 : rien de psychologique dans cette haine; il y a un homme de trop pour une même place.

${ }^{69}$ Voir encore, récemment, G. Zanker, qui voit dans la « générosité d'Ulysse » le couronnement de

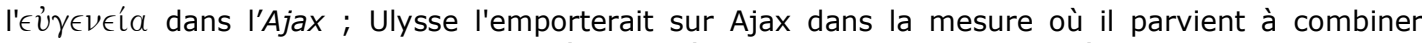
« le sens de la justice et le facteur de réponses émotionnelles comme la pitié... », « Sophocles' Ajax and the Heroic Values of the Iliad », Classical Quarterly, 42, 1992, p. 20-25 (p. 25).

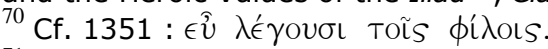

${ }^{71}$ Comme Jebb ou Moorhouse, Syntax of Sophocles, Leyde, 1982, p. 70 (« His worth prevails with me strongly as against his enmity »; voir déjà Schneidewin, Sophokles, I, $3^{e}$ éd., 1855, ad loc., qui glose l'accusatif en $\mathfrak{\epsilon}_{\mu} \mathrm{o}$ ) .
} 
pour la leçon d'un manuscrit unique (Paris. gr. 2735) ; $\pi \lambda^{\prime} \mathrm{o} \nu^{72}$. C'est, il me semble, manquer le but visé par Ulysse. Par l'ajout du $\mu \epsilon$, qui rend la proposition syntaxiquement difficile, il transforme une vérité qui pourrait se vouloir générale, où $\nu$ เ ã aurait un sens figuré, en une proposition beaucoup plus forte, dans laquelle il est question d'une défaite [405] personnelle. Il dit ainsi trois choses: que la valeur l'emporte sur l'inimitié, que cette affirmation vaut pour lui, et qu'il est prêt à accepter la défaite quand elle est de ce type. Une fois de plus, Ulysse présente à Agamemnon un monde plus complexe.

L'Atride, pas plus enclin qu'Ajax à une vision aussi relativiste, ne retient des propos d'Ulysse que le $\mu \epsilon$, qu'il retraduit, de manière rassurante, en une pluralité sur laquelle on peut porter un jugement de valeur généralisant sans remettre en cause l'existence d'une vérité absolue : «de tels hommes sont des mortels atteints d'incohérence ( $\left.\epsilon^{\prime \prime} \mu \pi \lambda \eta \kappa T O L\right) ~>^{73}$. Ulysse rebondit sur cette proposition (v. 1359). On peut y reconnaître l'affirmation de l'alternance et de I'instabilité, dans le domaine de la $\phi(\lambda i ́ a$, qu'avait déplorées Ajax en 679-82, et penser, comme Agamemnon ${ }^{74}$, qu'Ulysse fait référence au seul Ajax. En fait, la phrase, par l'absence même de réfèrent précis, prend une portée beaucoup plus large, dont le contexte lui-même permet de vérifier la vraisemblance : Agamemnon vient de traiter Ulysse de manière peu amène, alors qu'il avait fait de lui, peu de temps auparavant (1331), son plus grand ami : c'est la reconnaissance de cette impossible permanence qui amène Ulysse à blâmer les âmes rigides (1361) et, comme il l'avait fait à propos de la notion de défaite, à relativiser l'évaluation d'une même action, qui peut paraître lâche à Agamemnon et sembler pourtant juste aux yeux de tous les Grecs (1362 s.).

Ainsi, la défense de la dépouille d'Ajax se fait grâce à une argumentation qui nie ce pour quoi est mort le héros : l'identité immuable des êtres et des valeurs. On a surtout voulu y voir une immense capacité d'Ulysse à la sagesse et à la générosité, avec laquelle ne cadrait pas bien l'égoïsme que semble dénoter le vers 1367 («pour qui est-il naturel que je me donne de la peine sinon pour moi ? »). Stanford ${ }^{75}$ refuse de considérer l'intérêt personnel comme le véritable motif de la noble action d'Ulysse et préfère considérer la phrase comme une façon purement tactique de persuader définitivement Agamemnon, ainsi convaincu de servir les seuls intérêts d'Ulysse, son ami. En fait, Ulysse ne fait que redire ce qu'il avait déjà exprimé au début de la [406] pièce quand Athéna lui avait offert le pénible spectacle de la démence d'Ajax.

Le prologue donne en effet la clé qui permet d'accéder à la compréhension de la tragédie : à travers l'exemple d'Ajax, Athéna montre que I'homme ne peut prétendre à la permanence mais est voué aux renversements de fortune les plus soudains (cf. v. $131 \mathrm{~s}$.). Ulysse a bien enregistré la leçon : quand il plaint Ajax, c'est sur lui-même qu'il se lamente (v. 124) en prenant conscience que «nous tous qui vivons ne sommes rien que des fantômes ou une ombre légère » $(125 \mathrm{~s}$.$) . \epsilon$ l $\delta \omega \lambda$ ov et $\sigma \kappa \iota \alpha ́$ sont utilisés dans les poèmes homériques à propos des morts ${ }^{76}$. Le paradoxe qui consiste à comparer notre vie au quasinéant de la mort ${ }^{77}$ produit tous les autres : Ulysse reconnaît qu'aucune valeur n'est stable pour l'homme, et son discours reflète cette expérience. L'égocentrisme du vers 124, dont on fait l'expression de «l'égoïsme éclairé de

\footnotetext{
72 Sophocles, I, éd. et trad. par H. Lloyd-Jones, Cambridge (Mass.)-Londres (Loeb Classical Library); ad loc.

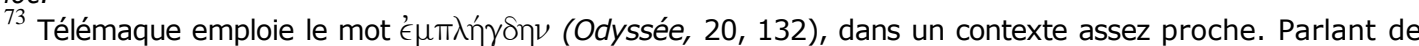
Pénélope, son fils l'accuse « d'honorer, bien qu'elle soit avisée, dans un mouvement d'incohérence, un mortel obscur et de refuser cet honneur au meilleur en le congédiant ». Le mot semble, dans les deux cas, désigner une incapacité à reconnaître les valeurs vraies, donc stables.

${ }^{74}$ Voir le vers 1360.

${ }^{75}$ Ad loc. ; il est suivi par Winnington-Ingram, p. 69, n. 34.

${ }^{76}$ Pour le premier, cf. Iliade XXIII, 72, 104; Odyssée 11, 476; pour le second, cf. Odyssée 10, 495 ; 11, 207; voir aussi Ajax, 1257.

${ }^{77}$ Cf. au vers 126 la place de $\zeta \omega \tilde{\omega} \mu \nu$, encadré par les deux termes.
} 
l'humanisme classique ${ }^{78}$, n'est pas à distinguer de celui qui a gêné au vers 1367 : dans un monde qui ne peut se référer à aucun absolu, le seul critère fixe est soi-même, et la sagesse consiste à adapter son action et son discours aux circonstances, comme le montre Ulysse dans son échange avec Agamemnon.

On a pu parler de I'humanisme d'Ulysse, de son humanité de héros classique, voire moderne, parce que le résultat de son discours est noble : Ajax sera enseveli. Il faut pourtant se garder d'un enthousiasme quelque peu anachronique. En glorifiant Ulysse comme on le fait, on confond la perspective de l'Histoire, peut-être même celle de l'homme-Sophocle (qui peuvent donner raison à Ulysse, plus « civilisé » en fin de compte qu'Ajax), avec celle de la tragédie. C'est sans doute pour cette raison que l'on se voit contraint de distinguer deux Ulysse : le cœur généreux de l'Ajax et le sophiste cynique et sans scrupule du Philoctète ${ }^{79}$. Le but, différent, puisqu'il s'agit de berner Philoctète pour s'emparer de son arc, implique des moyens différents, mais l'origine de l'action est la même : Ulysse obéit à l'injonction des dieux (cf. les vers 989 s.), et la règle qu'il s'impose demeure identique. À Philoctète qui le maudit Ulysse répond en effet : «je suis comme il faut être là où il faut, et quand il y a un concours d'hommes justes et nobles on ne trouvera personne de plus pieux que moi » [407] (v. 1049-1051). Cynique ou généreux selon les circonstances et la tâche à accomplir, Ulysse se soumet en fait à la loi divine, ce qui lui assure le salut, quel que soit le jugement que les hommes portent sur lui.

Cette attitude, aussi pragmatique que pieuse, se retrouve aussi bien dans l'Ajax que dans le Philoctète. L'humilité d'Ulysse n'est pas aussi tragique que le pense $\mathrm{Knox}^{80}$. Ulysse a su comprendre, contrairement à Ajax et à Agamemnon, que I'homme ne peut prétendre à l'absolu, réservé aux seuls dieux éternels. II accepte l'idée d'un kleos limité, mais il n'y renonce pas pour autant. Il est en effet le grand vainqueur de la tragédie. Outre qu'il garde les armes d'Achille, il obtient la reconnaissance implicite d'Athéna, à qui il a, par son discours et son attitude, montré qu'il avait assimilé la nécessité de relativiser toute notion humaine, y compris la haine, Agamemnon lui cède et il gagne l'admiration du chœur, qui ne lui était pourtant pas acquis, tant s'en faut (cf. les vers 1374 s.). Surmonter l'entêtement d'Agamemnon et obtenir l'ensevelissement d'Ajax feront partie de ses hauts faits, comme le reconnaît d'une certaine manière Agamemnon, sans prendre véritablement conscience de la portée des mots qu'il emploie au vers $1368^{81}$.

Qu'en est-il d'Ajax par contre ? Certes, il aura les honneurs du tombeau, mais son destin n'en reste pas moins terriblement tragique, dans la manière même dont il accède à cet honneur. C'est Ulysse, son pire ennemi, celui qui l'a dépouillé de son kleos en remportant les armes d'Achille, la cause donc de la catastrophe, qui lui restitue sa gloire. Cette gloire elle-même contredit tout ce pour quoi Ajax s'est battu : loin de correspondre à une action unanimement louée, à une excellence naturelle, elle n'est que le fruit d'une intervention ponctuelle, d'une décision humaine. Cette décision elle-même est le produit du seul discours, dont est banni de surcroît tout concept prétendant à la vérité absolue. Ainsi, grandeur et immortalisation du héros passent par la reconnaissance de son caractère dérisoire et éphémère. Ajax est nié pour la seconde fois, mais il est maintenant impuissant à réagir. Teucros, tout en exprimant sa gratitude, perçoit d'ailleurs ce qu'a d'impossible la situation du point de vue du mort et écarte Ulysse des préparatifs funèbres ${ }^{82}$.

[408] Loin d'être la fin heureuse que l'on se plaît à y voir, la dernière partie de

\footnotetext{
${ }^{78}$ Stanford, ad 124-6.

${ }^{79} \mathrm{Cf}$. ci-dessus, note 23.

${ }^{80}$ Op. cit., p. 26, 28.

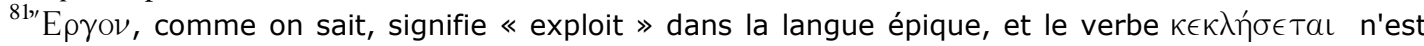
vraisemblablement pas utilisé au hasard par Sophocle.

${ }^{82}$ Voir les vers 1393-1395.
} 
l'Ajax redouble donc au contraire le caractère tragique de la pièce. La réconciliation, purement formelle el nullement unanime puisqu'elle n'atteint pas Agamemnon, laisse ouvert le problème de la place à donner à un individu de cette envergure dans le cadre de la communauté. Les Atrides ont mis en évidence, face à un Ajax dont la nature, toute hybristique qu'elle soit, servait singulièrement l'armée et méritait donc d'être reconnue, l'inanité d'une autorité absolue qui, faute de principes pertinents, se nie en voulant briser ce dont elle ne parvient pas à rendre compte. La solution d'Ulysse, qui compose avec les circonstances, accepte, de par son caractère atomistique, l'idée d'une grandeur toute relative et ne peut donc fournir au problème qu'une solution formelle. Du point de vue de l'intrigue elle-même, les choses ne finissent pas aussi bien qu'on le dit. Ajax, tel qu'il voulait être, meurt en effet pour la seconde fois. Le spectateur du $V^{e}$ siècle comprenait peut-être ainsi comment Ajax avait pu accéder au statut de héros athénien, mais l'essentiel pour l'auteur tragique qu'était Sophocle était de montrer le prix à payer pour une telle gloire. Le renom perpétuel repose sur l'acceptation par l'homme de son altérabilité et de sa légèreté, or Ajax s'est donné la mort précisément parce qu'il refusait d'adhérer à une telle conception du monde. La gloire, qui lui est imposée du dehors, n'est donc qu'un artefact, un effet de discours de surcroît ${ }^{83}$. Ulysse, lui, en offrant à Ajax une sépulture, évacue le problème qui a suscité la crise, celui de l'injustice de l'attribution des armes. Aucune ombre de scandale ne pèse plus sur lui : il devient désormais, sans contestation possible, le détenteur légitime des armes d'Achille. Nous sommes au comble de l'ironie tragique.

Fabienne Blaise,

Université Charles de Gaulle-Lille III.

\footnotetext{
${ }^{83}$ En fait. L'Ajax paraît bien moins archaïque et homérisant que ne l'affirmait Reinhardt (voir cidessus, p. 386) ; cette apparente recomposition du héros, qui repose en fait sur la négation de ce qu'il prétendait être, ainsi que l'utilisation tragique de l'agôn évoquent bien plutôt déjà le théâtre d'Euripide.
} 